\title{
Prediction of Phase State of Secondary Organic Aerosol Internally Mixed with Aqueous Inorganic Salts
}

Zechen Yu, Myoseon Jang*, and Azad Madhu

Department of Environmental Engineering Sciences, University of Florida, Gainesville, 32611, USA

Correspondence to: Myoseon Jang (mjang@ufl.com)

Number of sections: 3

Number of tables: 4

Number of figures: 6 
Section S1: Measurement of aerosol phase separation relative humidity (SRH).

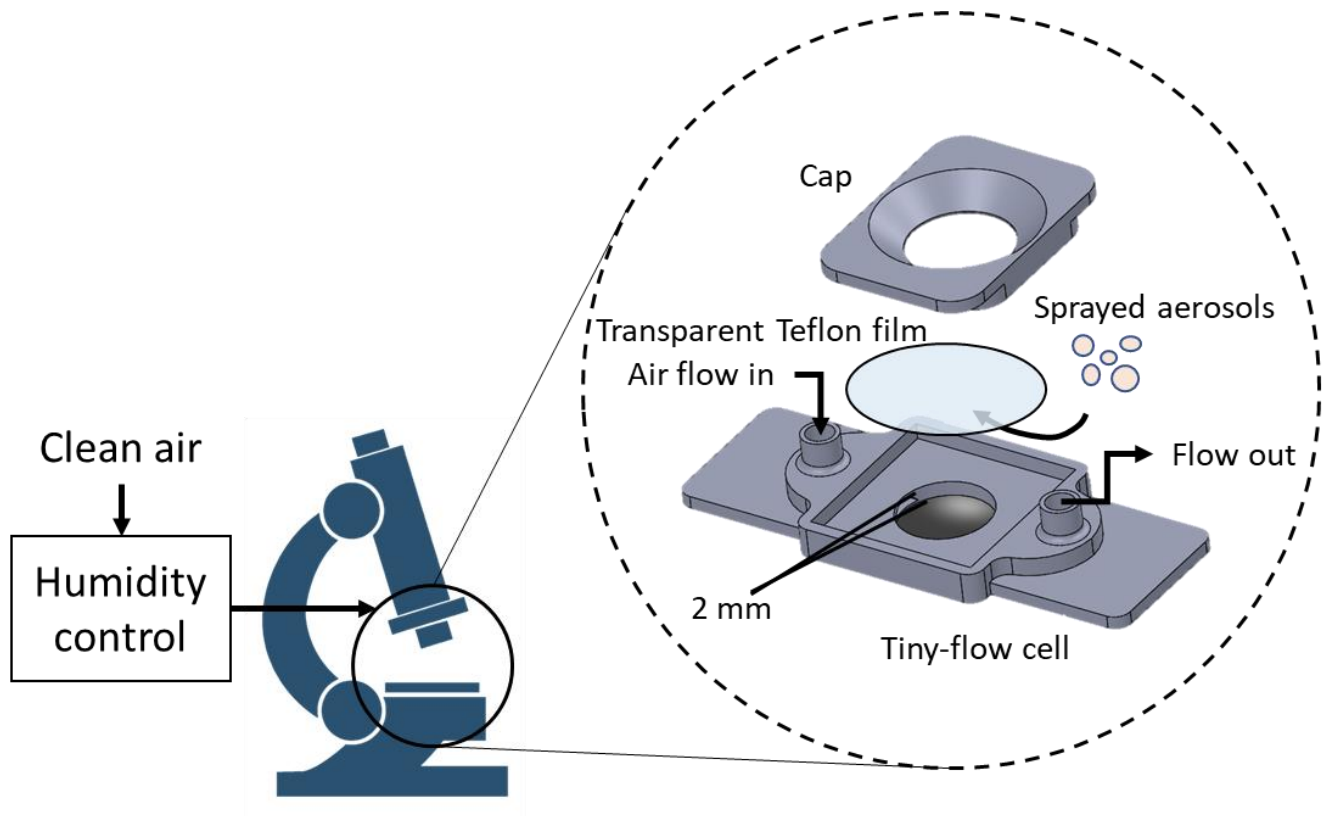

Figure S1. The experimental setup for the measurement of SRH using a flow cell and microscope.
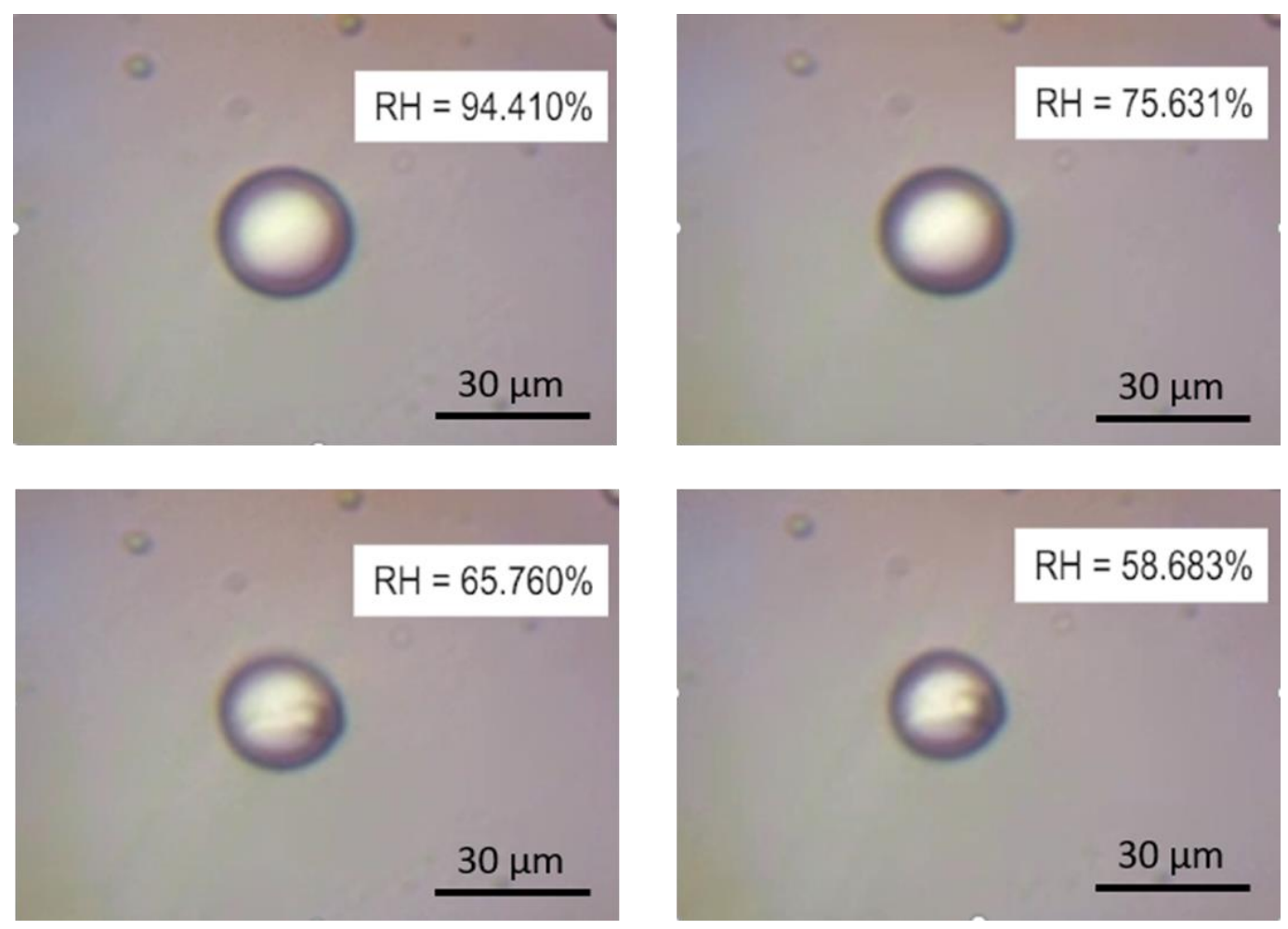

Figure S2. The optical image of the aerosol phase transition for the adipic acid in the presence of ammonium hydrogen sulfate. The $f_{\text {anion }}$ is 0.71 and the IOR is 1.98 . 
Table S1. Observations of phase separation relative humidity (SRH) of model organic species.

\begin{tabular}{|c|c|c|c|c|c|c|c|c|c|c|}
\hline Name & Formula & MW & $f_{\text {anion }}$ & $\mathrm{H}_{\text {bond }}$ & $\mathrm{O}: \mathrm{C}$ & $f_{\text {Nitrate }}$ & IOR & SRH & $\mathrm{ERH}^{\mathrm{a}}$ & Reff \\
\hline 1,2,6-hexanetriol & $\mathrm{C} 6 \mathrm{H} 14 \mathrm{O} 3$ & 134.2 & 0.50 & 3.00 & 0.50 & 0 & 0.51 & $77 \%$ & $38 \%$ & You et al. (2013) \\
\hline 1,2,6-hexanetriol & С6H14O3 & 134.2 & 0.50 & 3.00 & 0.50 & 1 & 0.80 & $0 \%$ & $7 \%$ & You et al. (2013) \\
\hline 1,2,6-hexanetriol & $\mathrm{C} 6 \mathrm{H} 14 \mathrm{O} 3$ & 134.2 & 0.67 & 3.00 & 0.50 & 0 & 0.58 & $0 \%$ & $4 \%$ & You et al. (2013) \\
\hline 2,2-dimethylsuccinic acid & $\mathrm{C} 6 \mathrm{H} 10 \mathrm{O} 4$ & 146.2 & 0.67 & 3.20 & 0.67 & 0 & 0.64 & $61 \%$ & $4 \%$ & You et al. (2013) \\
\hline 2,2-dimethylsuccinic acid & C6H10O4 & 146.2 & 0.67 & 3.20 & 0.67 & 0 & 2.54 & $61 \%$ & $4 \%$ & You et al. (2013) \\
\hline 2,2-dimethylsuccinic acid & $\mathrm{C} 6 \mathrm{H} 10 \mathrm{O} 4$ & 146.2 & 0.50 & 3.20 & 0.67 & 1 & 3.66 & $41 \%$ & $7 \%$ & You et al. (2013) \\
\hline 2,2-dimethylsuccinic acid & C6H10O4 & 146.2 & 0.50 & 3.20 & 0.67 & 1 & 0.96 & $40 \%$ & $7 \%$ & You et al. (2013) \\
\hline 2,2-dimethylsuccinic acid & C6H10O4 & 146.2 & 0.50 & 3.20 & 0.67 & 0 & 0.55 & $0 \%$ & $38 \%$ & You et al. (2013) \\
\hline 2,5-hexanediol & C6H14O2 & 118.2 & 0.50 & 2.00 & 0.33 & 0 & 0.45 & $89 \%$ & $38 \%$ & You et al. (2013) \\
\hline 2,5-hexanediol & $\mathrm{C} 6 \mathrm{H} 14 \mathrm{O} 2$ & 118.2 & 0.67 & 2.00 & 0.33 & 0 & 0.49 & $81 \%$ & $4 \%$ & You et al. (2013) \\
\hline 2,5-hexanediol & $\mathrm{C} 6 \mathrm{H} 14 \mathrm{O} 2$ & 118.2 & 0.50 & 2.00 & 0.33 & 1 & 0.70 & $64 \%$ & $7 \%$ & You et al. (2013) \\
\hline 2,5-hydroxybenzoic acid & $\mathrm{C} 7 \mathrm{H} 6 \mathrm{O} 4$ & 154.2 & 0.50 & 3.60 & 0.57 & 1 & 0.96 & $0 \%$ & $7 \%$ & You et al. (2013) \\
\hline 2,5-hydroxybenzoic acid & $\mathrm{C} 7 \mathrm{H} 6 \mathrm{O} 4$ & 154.2 & 0.50 & 3.60 & 0.57 & 1 & 3.86 & $0 \%$ & $7 \%$ & You et al. (2013) \\
\hline 2,5-hydroxybenzoic acid & C7H6O4 & 154.2 & 0.67 & 3.60 & 0.57 & 0 & 2.68 & $0 \%$ & $4 \%$ & You et al. (2013) \\
\hline 2,5-hydroxybenzoic acid & $\mathrm{C} 7 \mathrm{H} 6 \mathrm{O} 4$ & 154.2 & 0.67 & 3.60 & 0.57 & 0 & 0.71 & $0 \%$ & $4 \%$ & You et al. (2013) \\
\hline 2,5-hydroxybenzoic acid & $\mathrm{C} 7 \mathrm{H} 6 \mathrm{O} 4$ & 154.2 & 0.50 & 3.60 & 0.57 & 0 & 0.58 & $0 \%$ & $38 \%$ & You et al. (2013) \\
\hline 2-methylglutaric acid & C6H10O4 & 146.1 & 0.50 & 3.20 & 0.67 & 0 & 0.55 & $75 \%$ & $38 \%$ & You et al. (2013) \\
\hline 2-methylglutaric acid & C6H10O4 & 146.1 & 0.67 & 3.20 & 0.67 & 0 & 0.64 & $65 \%$ & $4 \%$ & You et al. (2013) \\
\hline 2-methylglutaric acid & C6H10O4 & 146.1 & 0.50 & 3.20 & 0.67 & 1 & 0.91 & $35 \%$ & $7 \%$ & You et al. (2013) \\
\hline 3,3-dimethylglutaric acid & $\mathrm{C} 7 \mathrm{H} 12 \mathrm{O} 4$ & 160.2 & 0.50 & 3.20 & 0.57 & 0 & 0.61 & $89 \%$ & $38 \%$ & You et al. (2013) \\
\hline 3,3-dimethylglutaric acid & $\mathrm{C} 7 \mathrm{H} 12 \mathrm{O} 4$ & 160.2 & 0.67 & 3.20 & 0.57 & 0 & 0.70 & $89 \%$ & $4 \%$ & You et al. (2013) \\
\hline 3,3-dimethylglutaric acid & $\mathrm{C} 7 \mathrm{H} 12 \mathrm{O} 4$ & 160.2 & 0.50 & 3.20 & 0.57 & 1 & 1.00 & $61 \%$ & $7 \%$ & You et al. (2013) \\
\hline Citric acid & C6H8O7 & 192.1 & 0.50 & 5.80 & 1.17 & 1 & 1.26 & $0 \%$ & $7 \%$ & You et al. (2013) \\
\hline Citric acid & C6H8O7 & 192.1 & 0.67 & 5.80 & 1.17 & 0 & 0.84 & $0 \%$ & $4 \%$ & You et al. (2013) \\
\hline Citric acid & C6H8O7 & 192.1 & 0.50 & 5.80 & 1.17 & 0 & 0.73 & $0 \%$ & $38 \%$ & You et al. (2013) \\
\hline Diethyl sebacate & $\mathrm{C} 14 \mathrm{H} 26 \mathrm{O} 4$ & 258.4 & 0.50 & 0.00 & 0.29 & 1 & 1.62 & $100 \%$ & $7 \%$ & You et al. (2013) \\
\hline Diethyl sebacate & $\mathrm{C} 14 \mathrm{H} 26 \mathrm{O} 4$ & 258.4 & 0.67 & 0.00 & 0.29 & 0 & 1.12 & $100 \%$ & $4 \%$ & You et al. (2013) \\
\hline Diethyl sebacate & $\mathrm{C} 14 \mathrm{H} 26 \mathrm{O} 4$ & 258.4 & 0.50 & 0.00 & 0.29 & 0 & 0.98 & $100 \%$ & $38 \%$ & You et al. (2013) \\
\hline
\end{tabular}




\begin{tabular}{|c|c|c|c|c|c|c|c|c|c|c|}
\hline Name & Formula & MW & $f_{\text {anion }}$ & $\mathrm{H}_{\text {bond }}$ & $\mathrm{O}: \mathrm{C}$ & $f_{\text {Nitrate }}$ & IOR & SRH & $\mathrm{ERH}^{\mathrm{a}}$ & Reff \\
\hline Diethyl-L-tartrate & C8H14O6 & 206.2 & 0.50 & 2.00 & 0.75 & 0 & 0.74 & $90 \%$ & $38 \%$ & You et al. (2013) \\
\hline Diethyl-L-tartrate & C8H14O6 & 206.2 & 0.67 & 2.00 & 0.75 & 0 & 0.85 & $65 \%$ & $4 \%$ & You et al. (2013) \\
\hline Diethyl-L-tartrate & C8H14O6 & 206.2 & 0.50 & 2.00 & 0.75 & 1 & 1.29 & $29 \%$ & $7 \%$ & You et al. (2013) \\
\hline Diethylmalonic acid & C7H12O4 & 160.2 & 0.50 & 3.20 & 0.57 & 0 & 0.61 & $89 \%$ & $38 \%$ & You et al. (2013) \\
\hline Diethylmalonic acid & C7H12O4 & 160.2 & 0.67 & 3.20 & 0.57 & 0 & 0.70 & $88 \%$ & $4 \%$ & You et al. (2013) \\
\hline Diethylmalonic acid & C7H12O4 & 160.2 & 0.50 & 3.20 & 0.57 & 1 & 0.95 & $74 \%$ & $7 \%$ & You et al. (2013) \\
\hline Glutaric acid & C5H8O4 & 132.1 & 0.50 & 3.20 & 0.80 & 1 & 0.83 & $0 \%$ & $7 \%$ & You et al. (2013) \\
\hline Glutaric acid & C5H8O4 & 132.1 & 0.67 & 3.20 & 0.80 & 0 & 0.60 & $0 \%$ & $4 \%$ & You et al. (2013) \\
\hline Glutaric acid & C5H8O4 & 132.1 & 0.50 & 3.20 & 0.80 & 0 & 0.50 & $0 \%$ & $38 \%$ & You et al. (2013) \\
\hline Glycerol & С3H8O3 & 92.1 & 0.50 & 3.00 & 1.00 & 1 & 0.55 & $0 \%$ & $7 \%$ & You et al. (2013) \\
\hline Glycerol & C3H8O3 & 92.1 & 0.67 & 3.00 & 1.00 & 0 & 0.42 & $0 \%$ & $4 \%$ & You et al. (2013) \\
\hline Glycerol & С3H8O3 & 92.1 & 0.50 & 3.00 & 1.00 & 0 & 0.35 & $0 \%$ & $38 \%$ & You et al. (2013) \\
\hline Levoglucosan & C6H10O5 & 162.1 & 0.50 & 3.00 & 0.83 & 1 & 1.07 & $0 \%$ & $7 \%$ & You et al. (2013) \\
\hline Levoglucosan & C6H10O5 & 162.1 & 0.67 & 3.00 & 0.83 & 0 & 0.74 & $0 \%$ & $4 \%$ & You et al. (2013) \\
\hline Levoglucosan & C6H10O5 & 162.1 & 0.50 & 3.00 & 0.83 & 0 & 0.61 & $0 \%$ & $38 \%$ & You et al. (2013) \\
\hline Maleic acid & $\mathrm{C} 4 \mathrm{H} 4 \mathrm{O} 4$ & 116.1 & 0.50 & 3.20 & 1.00 & 1 & 0.76 & $0 \%$ & $7 \%$ & You et al. (2013) \\
\hline Maleic acid & C4H4O4 & 116.1 & 0.67 & 3.20 & 1.00 & 0 & 0.50 & $0 \%$ & $4 \%$ & You et al. (2013) \\
\hline Maleic acid & C4H4O4 & 116.1 & 0.50 & 3.20 & 1.00 & 0 & 0.44 & $0 \%$ & $38 \%$ & You et al. (2013) \\
\hline Malic acid & C4H6O5 & 134.9 & 0.50 & 4.20 & 1.25 & 1 & 0.89 & $0 \%$ & $7 \%$ & You et al. (2013) \\
\hline Malic acid & C4H6O5 & 134.9 & 0.67 & 4.20 & 1.25 & 0 & 0.62 & $0 \%$ & $4 \%$ & You et al. (2013) \\
\hline Malic acid & $\mathrm{C} 4 \mathrm{H} 6 \mathrm{O} 5$ & 134.9 & 0.50 & 4.20 & 1.25 & 0 & 0.49 & $0 \%$ & $38 \%$ & You et al. (2013) \\
\hline Malonic acid & $\mathrm{C} 3 \mathrm{H} 4 \mathrm{O} 4$ & 104.1 & 0.50 & 3.20 & 1.33 & 1 & 0.65 & $0 \%$ & $7 \%$ & You et al. (2013) \\
\hline Malonic acid & $\mathrm{C} 3 \mathrm{H} 4 \mathrm{O} 4$ & 104.1 & 0.67 & 3.20 & 1.33 & 0 & 0.45 & $0 \%$ & $4 \%$ & You et al. (2013) \\
\hline Malonic acid & $\mathrm{C} 3 \mathrm{H} 4 \mathrm{O} 4$ & 104.1 & 0.50 & 3.20 & 1.33 & 0 & 0.39 & $0 \%$ & $38 \%$ & You et al. (2013) \\
\hline Suberic acid monomethyl ester & C9H16O4 & 188.2 & 0.50 & 1.60 & 0.44 & 1 & 1.12 & $100 \%$ & $7 \%$ & You et al. (2013) \\
\hline Suberic acid monomethyl ester & C9H16O4 & 188.2 & 0.67 & 1.60 & 0.44 & 0 & 0.82 & $100 \%$ & $4 \%$ & You et al. (2013) \\
\hline Suberic acid monomethyl ester & C9H16O4 & 188.2 & 0.50 & 1.60 & 0.44 & 0 & 0.71 & $100 \%$ & $38 \%$ & You et al. (2013) \\
\hline
\end{tabular}




\begin{tabular}{|c|c|c|c|c|c|c|c|c|c|c|}
\hline Name & Formula & MW & $f_{\text {anion }}$ & $\mathrm{H}_{\text {bond }}$ & $\mathrm{O}: \mathrm{C}$ & $f_{\text {Nitrate }}$ & IOR & SRH & $\mathrm{ERH}^{\mathrm{a}}$ & Reff \\
\hline$\alpha, 4$-dihydroxy-3-methoxybenzeneacetic acid & C9H10O5 & 198.2 & 0.50 & 3.60 & 0.56 & 0 & 0.75 & $73 \%$ & $38 \%$ & You et al. (2013) \\
\hline$\alpha, 4$-dihydroxy-3-methoxybenzeneacetic acid & C9H10O5 & 198.2 & 0.67 & 3.60 & 0.56 & 0 & 3.45 & $46 \%$ & $4 \%$ & You et al. (2013) \\
\hline$\alpha, 4$-dihydroxy-3-methoxybenzeneacetic acid & C9H10O5 & 198.2 & 0.67 & 3.60 & 0.56 & 0 & 0.86 & $38 \%$ & $4 \%$ & You et al. (2013) \\
\hline$\alpha, 4$-dihydroxy-3-methoxybenzeneacetic acid & C9H10O5 & 198.2 & 0.50 & 3.60 & 0.56 & 1 & 1.30 & $0 \%$ & $7 \%$ & You et al. (2013) \\
\hline$\alpha, 4$-dihydroxy-3-methoxybenzeneacetic acid & C9H10O5 & 198.2 & 0.50 & 3.60 & 0.56 & 1 & 4.96 & $0 \%$ & $7 \%$ & You et al. (2013) \\
\hline 1,2,6-hexanetriol & C6H14O3 & 134.2 & 0.50 & 3.00 & 0.50 & 0 & 0.66 & $73 \%$ & $38 \%$ & Bertram et al. (2011) \\
\hline 1,2,6-hexanetriol & C6H14O3 & 134.2 & 0.50 & 3.00 & 0.50 & 0 & 0.25 & $72 \%$ & $38 \%$ & Bertram et al. (2011) \\
\hline 1,2,6-hexanetriol & C6H14O3 & 134.2 & 0.50 & 3.00 & 0.50 & 0 & 3.94 & $70 \%$ & $38 \%$ & Bertram et al. (2011) \\
\hline 1,2,6-hexanetriol & C6H14O3 & 134.2 & 0.50 & 3.00 & 0.50 & 0 & 1.48 & $69 \%$ & $38 \%$ & Bertram et al. (2011) \\
\hline 2,2-dimethylsuccinic acid & C6H10O4 & 146.2 & 0.50 & 3.20 & 0.67 & 0 & 3.69 & $64 \%$ & $38 \%$ & Bertram et al. (2011) \\
\hline 2,2-dimethylsuccinic acid & C6H10O4 & 146.2 & 0.50 & 3.20 & 0.67 & 0 & 2.22 & $62 \%$ & $38 \%$ & Bertram et al. (2011) \\
\hline 2,2-dimethylsuccinic acid & C6H10O4 & 146.2 & 0.50 & 3.20 & 0.67 & 0 & 0.74 & $0 \%$ & $38 \%$ & Bertram et al. (2011) \\
\hline 2,2-dimethylsuccinic acid & C6H10O4 & 146.2 & 0.50 & 3.20 & 0.67 & 0 & 0.92 & $0 \%$ & $38 \%$ & Bertram et al. (2011) \\
\hline 2,2-dimethylsuccinic acid & C6H10O4 & 146.2 & 0.50 & 3.20 & 0.67 & 0 & 1.11 & $0 \%$ & $38 \%$ & Bertram et al. (2011) \\
\hline 2,5-hydroxybenzoic acid & C7H6O4 & 154.2 & 0.50 & 3.60 & 0.57 & 0 & 3.89 & $64 \%$ & $38 \%$ & Bertram et al. (2011) \\
\hline 2,5-hydroxybenzoic acid & C7H6O4 & 154.2 & 0.50 & 3.60 & 0.57 & 0 & 2.34 & $63 \%$ & $38 \%$ & Bertram et al. (2011) \\
\hline 2,5-hydroxybenzoic acid & $\mathrm{C} 7 \mathrm{H} 6 \mathrm{O} 4$ & 154.2 & 0.50 & 3.60 & 0.57 & 0 & 5.84 & $62 \%$ & $38 \%$ & Bertram et al. (2011) \\
\hline 2,5-hydroxybenzoic acid & C7H6O4 & 154.2 & 0.50 & 3.60 & 0.57 & 0 & 1.46 & $0 \%$ & $38 \%$ & Bertram et al. (2011) \\
\hline 2,5-hydroxybenzoic acid & $\mathrm{C} 7 \mathrm{H} 6 \mathrm{O} 4$ & 154.2 & 0.50 & 3.60 & 0.57 & 0 & 1.17 & $0 \%$ & $38 \%$ & Bertram et al. (2011) \\
\hline 2,5-hydroxybenzoic acid & C7H6O4 & 154.2 & 0.50 & 3.60 & 0.57 & 0 & 0.78 & $0 \%$ & $38 \%$ & Bertram et al. (2011) \\
\hline Diethyl sebacate & $\mathrm{C} 14 \mathrm{H} 26 \mathrm{O} 4$ & 258.4 & 0.50 & 0.00 & 0.29 & 0 & 1.87 & $100 \%$ & $38 \%$ & Bertram et al. (2011) \\
\hline Diethyl sebacate & $\mathrm{C} 14 \mathrm{H} 26 \mathrm{O} 4$ & 258.4 & 0.50 & 0.00 & 0.29 & 0 & 0.61 & $100 \%$ & $38 \%$ & Bertram et al. (2011) \\
\hline Diethyl sebacate & $\mathrm{C} 14 \mathrm{H} 26 \mathrm{O} 4$ & 258.4 & 0.50 & 0.00 & 0.29 & 0 & 0.34 & $100 \%$ & $38 \%$ & Bertram et al. (2011) \\
\hline Diethyl sebacate & C14H26O4 & 258.4 & 0.50 & 0.00 & 0.29 & 0 & 5.59 & $98 \%$ & $38 \%$ & Bertram et al. (2011) \\
\hline
\end{tabular}

\footnotetext{
${ }^{a}$ The ERH is estimated using the neural network model as described in Section S2.
} 


\begin{tabular}{|c|c|c|c|c|c|c|c|c|c|c|}
\hline Name & Formula & MW & $f_{\text {anion }}$ & $\mathrm{H}_{\text {bond }}$ & $\mathrm{O}: \mathrm{C}$ & $f_{\text {Nitrate }}$ & IOR & SRH & $\mathrm{ERH}^{\mathrm{a}}$ & Reff \\
\hline Diethyl sebacate & $\mathrm{C} 14 \mathrm{H} 26 \mathrm{O} 4$ & 258.4 & 0.50 & 0.00 & 0.29 & 0 & 10.82 & $0 \%$ & $38 \%$ & Bertram et al. (2011) \\
\hline Suberic acid monomethyl ester & С9H16O4 & 188.2 & 0.50 & 1.60 & 0.44 & 0 & 4.11 & $100 \%$ & $38 \%$ & Bertram et al. (2011) \\
\hline Suberic acid monomethyl ester & C9H16O4 & 188.2 & 0.50 & 1.60 & 0.44 & 0 & 1.40 & $100 \%$ & $38 \%$ & Bertram et al. (2011) \\
\hline Suberic acid monomethyl ester & С9H16O4 & 188.2 & 0.50 & 1.60 & 0.44 & 0 & 0.86 & $100 \%$ & $38 \%$ & Bertram et al. (2011) \\
\hline Suberic acid monomethyl ester & С9H16O4 & 188.2 & 0.50 & 1.60 & 0.44 & 0 & 0.44 & $100 \%$ & $38 \%$ & Bertram et al. (2011) \\
\hline$\alpha, 4$-dihydroxy-3-methoxybenzeneacetic acid & C9H10O5 & 198.2 & 0.50 & 3.60 & 0.56 & 0 & 3.75 & $80 \%$ & $38 \%$ & Bertram et al. (2011) \\
\hline$\alpha, 4$-dihydroxy-3-methoxybenzeneacetic acid & C9H10O5 & 198.2 & 0.50 & 3.60 & 0.56 & 0 & 0.25 & $0 \%$ & $38 \%$ & Bertram et al. (2011) \\
\hline 1,2-hexanediol & $\mathrm{C} 6 \mathrm{H} 14 \mathrm{O} 2$ & 118.2 & 0.50 & 2.00 & 0.33 & 0 & 0.90 & $95 \%$ & $38 \%$ & Marcolli et al., (2006) \\
\hline 1,2-hexanediol & $\mathrm{C} 6 \mathrm{H} 14 \mathrm{O} 2$ & 118.2 & 0.50 & 2.00 & 0.33 & 1 & 1.48 & $71 \%$ & $7 \%$ & Marcolli et al., (2006) \\
\hline 1,4-butanediol & $\mathrm{C} 4 \mathrm{H} 10 \mathrm{O} 2$ & 90.1 & 0.50 & 2.00 & 0.50 & 0 & 0.68 & $80 \%$ & $38 \%$ & Marcolli et al., (2006) \\
\hline 1,4-butanediol & $\mathrm{C} 4 \mathrm{H} 10 \mathrm{O} 2$ & 90.1 & 0.50 & 2.00 & 0.50 & 1 & 1.13 & $0 \%$ & $7 \%$ & Marcolli et al., (2006) \\
\hline Glucose & C6H12O6 & 180.2 & 0.50 & 5.00 & 1.00 & 0 & 0.68 & $0 \%$ & $38 \%$ & Marcolli et al., (2006) \\
\hline$\alpha, 4$-dihydroxy-3-methoxybenzeneacetic acid & C9H10O5 & 198.2 & 0.50 & 3.60 & 0.56 & 0 & 1.50 & $81 \%$ & $38 \%$ & O'Brien et al. (2015) \\
\hline 2-methylglutaric acid & C6H10O4 & 146.1 & 0.50 & 3.2 & 0.67 & 0 & 1.00 & $79 \%$ & $38 \%$ & Losey et al. (2018) \\
\hline 2-methylglutaric acid & C6H10O4 & 146.1 & 0.57 & 3.2 & 0.67 & 0 & 1.00 & $72 \%$ & $23 \%$ & Losey et al. (2018) \\
\hline 2-methylglutaric acid & C6H10O4 & 146.1 & 0.67 & 3.2 & 0.67 & 0 & 1.00 & $63 \%$ & $4 \%$ & Losey et al. (2018) \\
\hline 2-methylglutaric acid & C6H10O4 & 146.1 & 1 & 3.2 & 0.67 & 0 & 1.00 & $34 \%$ & $0 \%$ & Losey et al. (2018) \\
\hline 3-methylglutaric acid & C6H10O4 & 146.1 & 0.50 & 3.2 & 0.67 & 0 & 1.00 & $73 \%$ & $38 \%$ & Losey et al. (2018) \\
\hline 3-methylglutaric acid & C6H10O4 & 146.1 & 0.57 & 3.2 & 0.67 & 0 & 1.00 & $69 \%$ & $23 \%$ & Losey et al. (2018) \\
\hline 3-methylglutaric acid & C6H10O4 & 146.1 & 0.67 & 3.2 & 0.67 & 0 & 1.00 & $64 \%$ & $4 \%$ & Losey et al. (2018) \\
\hline 3-methylglutaric acid & C6H10O4 & 146.1 & 1 & 3.2 & 0.67 & 0 & 1.00 & $0 \%$ & $0 \%$ & Losey et al. (2018) \\
\hline 2.2-Bis(hydroxymethyl)butyric acid & C6H12O4 & 148.2 & 0.50 & 3.6 & 0.67 & 0 & 1.50 & $48 \%$ & $38 \%$ & Losey et al. (2018) \\
\hline 2.2-Bis(hydroxymethyl)butyric acid & C6H12O4 & 148.2 & 0.57 & 3.6 & 0.67 & 0 & 1.50 & $46 \%$ & $23 \%$ & Losey et al. (2018) \\
\hline 2.2-Bis(hydroxymethyl)butyric acid & C6H12O4 & 148.2 & 0.67 & 3.6 & 0.67 & 0 & 1.50 & $32 \%$ & $4 \%$ & Losey et al. (2018) \\
\hline 2.2-Bis(hydroxymethyl)butyric acid & $\mathrm{C} 6 \mathrm{H} 12 \mathrm{O} 4$ & 148.2 & 1.00 & 3.6 & 0.67 & 0 & 1.50 & $0 \%$ & $0 \%$ & Losey et al. (2018) \\
\hline
\end{tabular}

${ }^{a}$ The ERH is estimated using the neural network model as described in Section S2. 


\begin{tabular}{|c|c|c|c|c|c|c|c|c|c|c|}
\hline Name & Formula & MW & $f_{\text {anion }}$ & $\mathrm{H}_{\text {bond }}$ & $\mathrm{O}: \mathrm{C}$ & $f_{\text {Nitrate }}$ & IOR & SRH & $\mathrm{ERH}^{\mathrm{a}}$ & Reff \\
\hline 3,3-dimethylglutaric acid & $\mathrm{C} 7 \mathrm{H} 12 \mathrm{O} 4$ & 160.2 & 0.50 & 3.20 & 0.57 & 0 & 1.00 & $90 \%$ & $38 \%$ & Losey et al. (2018) \\
\hline 3,3-dimethylglutaric acid & $\mathrm{C} 7 \mathrm{H} 12 \mathrm{O} 4$ & 160.2 & 0.57 & 3.20 & 0.57 & 0 & 1.00 & $88 \%$ & $23 \%$ & Losey et al. (2018) \\
\hline 3,3-dimethylglutaric acid & $\mathrm{C} 7 \mathrm{H} 12 \mathrm{O} 4$ & 160.2 & 0.67 & 3.20 & 0.57 & 0 & 1.00 & $87 \%$ & $4 \%$ & Losey et al. (2018) \\
\hline 3,3-dimethylglutaric acid & $\mathrm{C} 7 \mathrm{H} 12 \mathrm{O} 4$ & 160.2 & 1.00 & 3.20 & 0.57 & 0 & 1.00 & $100 \%$ & $0 \%$ & Losey et al. (2018) \\
\hline Diethylmalonic acid & $\mathrm{C} 7 \mathrm{H} 12 \mathrm{O} 4$ & 160.2 & 0.50 & 3.20 & 0.57 & 0 & 1.00 & $94 \%$ & $38 \%$ & Losey et al. (2018) \\
\hline Diethylmalonic acid & $\mathrm{C} 7 \mathrm{H} 12 \mathrm{O} 4$ & 160.2 & 0.57 & 3.20 & 0.57 & 0 & 1.00 & $93 \%$ & $23 \%$ & Losey et al. (2018) \\
\hline Diethylmalonic acid & $\mathrm{C} 7 \mathrm{H} 12 \mathrm{O} 4$ & 160.2 & 0.67 & 3.20 & 0.57 & 0 & 1.00 & $92 \%$ & $4 \%$ & Losey et al. (2018) \\
\hline Diethylmalonic acid & $\mathrm{C} 7 \mathrm{H} 12 \mathrm{O} 4$ & 160.2 & 1.00 & 3.20 & 0.57 & 0 & 1.00 & $100 \%$ & $0 \%$ & Losey et al. (2018) \\
\hline 1,2,6-hexanetriol & C6H14O3 & 134.2 & 0.50 & 3.00 & 0.50 & 0 & 1.00 & $79 \%$ & $38 \%$ & Losey et al. (2018) \\
\hline 1,2,6-hexanetriol & $\mathrm{C} 6 \mathrm{H} 14 \mathrm{O} 3$ & 134.2 & 0.57 & 3.00 & 0.50 & 0 & 1.00 & $67 \%$ & $23 \%$ & Losey et al. (2018) \\
\hline 1,2,6-hexanetriol & C6H14O3 & 134.2 & 0.67 & 3.00 & 0.50 & 0 & 1.00 & $39 \%$ & $4 \%$ & Losey et al. (2018) \\
\hline 1,2,6-hexanetriol & C6H14O3 & 134.2 & 1.00 & 3.00 & 0.50 & 0 & 1.00 & $0 \%$ & $0 \%$ & Losey et al. (2018) \\
\hline 1,4-butanediol & $\mathrm{C} 4 \mathrm{H} 10 \mathrm{O} 2$ & 90 & 1.00 & 2.00 & 0.50 & 0 & 0.94 & $0 \%$ & $0 \%$ & This study \\
\hline 1,4-butanediol & $\mathrm{C} 4 \mathrm{H} 10 \mathrm{O} 2$ & 90 & 0.50 & 2.00 & 0.50 & 0 & 1.90 & $62.3 \%$ & $38 \%$ & This study \\
\hline adipic acid & C6H10O4 & 146 & 0.50 & 3.20 & 0.67 & 0 & 1.92 & $90.0 \%$ & $38 \%$ & This study \\
\hline adipic acid & C6H10O4 & 146 & 1.00 & 3.20 & 0.67 & 0 & 1.96 & $51.3 \%$ & $0 \%$ & This study \\
\hline adipic acid & C6H10O4 & 146 & 0.71 & 3.20 & 0.67 & 0 & 1.98 & $69.2 \%$ & $0 \%$ & This study \\
\hline adipic acid & C6H10O4 & 146 & 1.00 & 3.20 & 0.67 & 0 & 2.08 & $54.0 \%$ & $0 \%$ & This study \\
\hline adipic acid & C6H10O4 & 146 & 0.84 & 3.20 & 0.67 & 0 & 1.98 & $57.7 \%$ & $0 \%$ & This study \\
\hline adipic acid & C6H10O4 & 146 & 0.91 & 3.20 & 0.67 & 0 & 1.94 & $57.4 \%$ & $0 \%$ & This study \\
\hline adipic acid & C6H10O4 & 146 & 0.55 & 3.20 & 0.67 & 0 & 2.00 & $79.5 \%$ & $28 \%$ & This study \\
\hline adipic acid & C6H10O4 & 146 & 0.63 & 3.20 & 0.67 & 0 & 2.00 & $72.0 \%$ & $11 \%$ & This study \\
\hline azelaic Acid & С9H16O4 & 152 & 0.50 & 3.20 & 0.44 & 0 & 1.92 & $>95 \%$ & $38 \%$ & This study \\
\hline azelaic Acid & $\mathrm{C} 9 \mathrm{H} 16 \mathrm{O} 4$ & 152 & 0.67 & 3.20 & 0.44 & 0 & 0.56 & $91.7 \%$ & $4 \%$ & This study \\
\hline azelaic Acid & $\mathrm{C} 9 \mathrm{H} 16 \mathrm{O} 4$ & 152 & 0.86 & 3.20 & 0.44 & 0 & 1.95 & $88.0 \%$ & $0 \%$ & This study \\
\hline mesaconic acid & C5H6O4 & 130 & 0.50 & 3.20 & 0.80 & 0 & 2.07 & $53.0 \%$ & $38 \%$ & This study \\
\hline mesaconic acid & C5H6O4 & 130 & 0.59 & 3.20 & 0.80 & 0 & 1.92 & $0.0 \%$ & $20 \%$ & This study \\
\hline
\end{tabular}

a The ERH is estimated using the neural network model as described in Section S2. 
27 Table S1. (continued)

\begin{tabular}{ccccccccccc}
\hline Name & Formula & MW & $f_{\text {anion }}$ & $\mathrm{H}_{\text {bond }}$ & O:C & $f_{\text {Nitrate }}$ & IOR & SRH & ERH $^{\text {a }}$ & Reff \\
\hline mesaconic acid & C5H6O4 & 130 & 0.67 & 3.20 & 0.80 & 0 & 0.97 & $0.0 \%$ & $4 \%$ & This study \\
suberic acid & C8H14O4 & 174 & 0.50 & 3.20 & 0.50 & 0 & 2.02 & $>95 \%$ & $38 \%$ & This study \\
suberic acid & C8H14O4 & 174 & 0.67 & 3.20 & 0.50 & 0 & 2.11 & $78.3 \%$ & $4 \%$ & This study \\
suberic acid & C8H14O4 & 174 & 0.80 & 3.20 & 0.50 & 0 & 1.97 & $76.7 \%$ & $0 \%$ & This study \\
suberic acid & C8H14O4 & 174 & 1.00 & 3.20 & 0.50 & 0 & 0.50 & $75.3 \%$ & $0 \%$ & This study \\
\hline
\end{tabular}

${ }^{\mathrm{a}}$ The ERH is estimated using the neural network model as described in Section S2. 

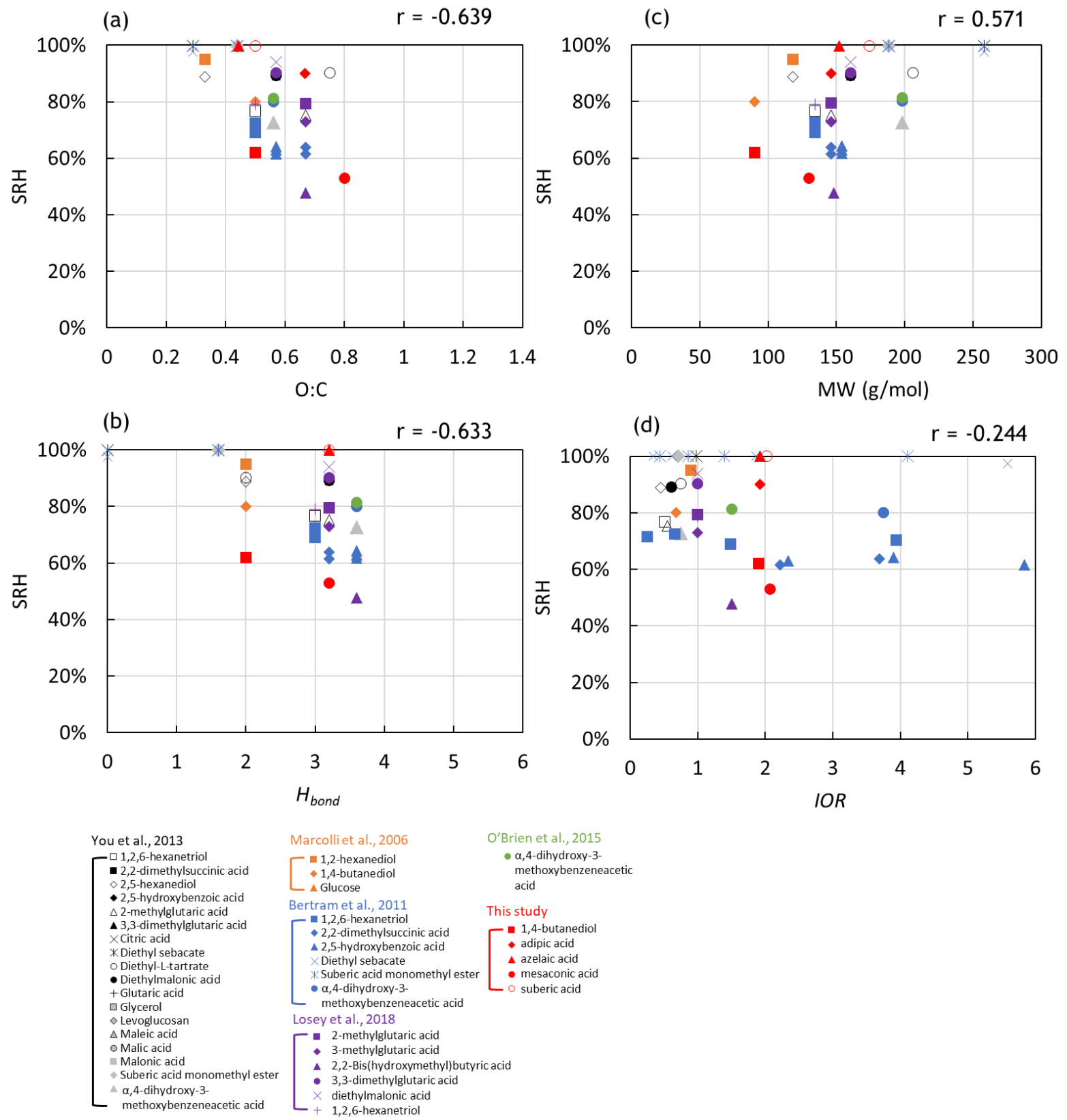

31 Figure S3. The observations of SRH vs. (a) O:C ratio; (b) hydrogen bond parameter $\left(H_{\text {bond }}\right)$; (c)

$32 \mathrm{MW}(\mathrm{g} / \mathrm{mol})$; and $(\mathrm{d})$ inorganic to organic dry mass ratio $(I O R)$ for the cases which contain

33 ammonium sulfate only. Total 41 cases are plotted. 

salts.

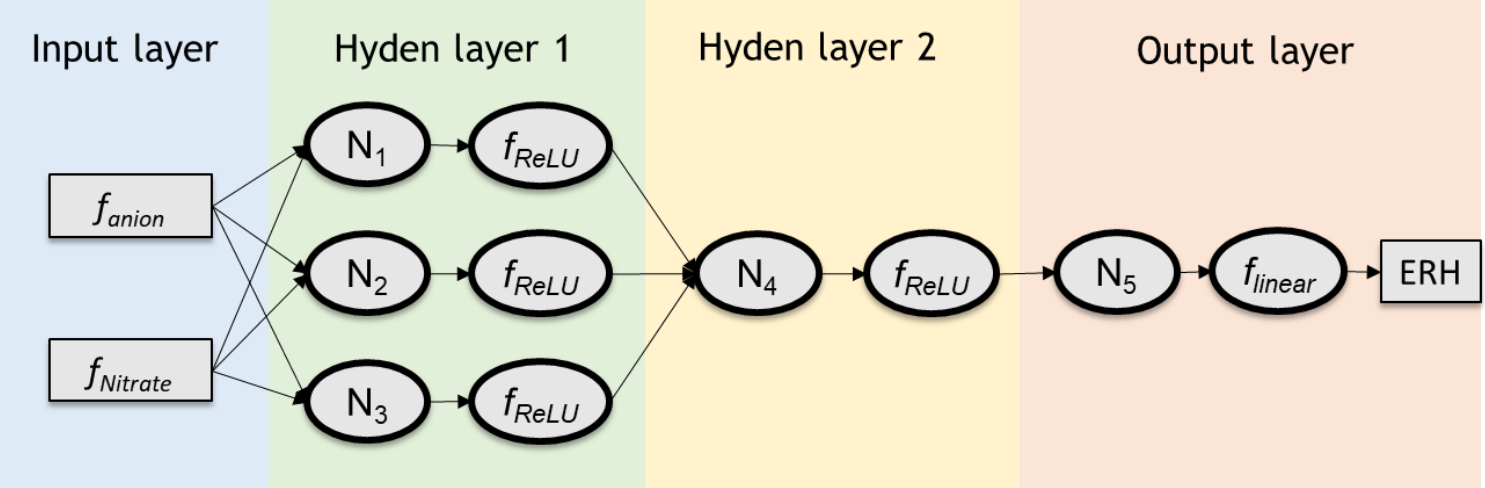

$$
N_{i}=\sum \text { weight }_{j} \times \operatorname{lnput} t_{j}+\text { bias }_{i}(\text { for } i=1-5)
$$

42

43

Table S2. Model parameters derived from deep neural network for the prediction of ERH

\begin{tabular}{c|l|c}
\hline $\begin{array}{c}\text { Neuron } \\
\#\end{array}$ & \multicolumn{1}{|c}{ Weighted sum } & $\begin{array}{c}\text { Activation } \\
\text { function }\end{array}$ \\
\hline $\mathrm{N}_{1}$ & $\mathrm{~N}_{1}=1.54463 \times f_{\text {anion }}-0.92430 \times f_{\text {nitrate }}-0.073745$ & \\
$\mathrm{~N}_{2}$ & $\mathrm{~N}_{2}=-0.63382 \times f_{\text {anion }}+0.82856 \times f_{\text {nitrate }}+0.288342$ & $\begin{array}{l}\text { ReLU }(\mathrm{x})= \\
0,(\mathrm{x}<0) \\
\mathrm{x},(\mathrm{x} \geq 0)\end{array}$ \\
$\mathrm{N}_{3}$ & $\mathrm{~N}_{3}=-0.18594 \times f_{\text {anion }}+0.63382 \times f_{\text {nitrate }}+0.366726$ & \\
$\mathrm{~N}_{4}$ & $\mathrm{~N}_{4}=-0.50581 \times \mathrm{N}_{1}-1.15781 \times \mathrm{N}_{2}+0.68805 \times \mathrm{N}_{3}+0.33499$ & \\
\hline $\mathrm{N}_{5}$ & $\mathrm{~N}_{5}=2.21228 \times \mathrm{N}_{4}+0.00018$ & Linear $(\mathrm{x})=\mathrm{x}$ \\
\hline
\end{tabular}

Figure S4. Diagram of neural network used to predict efflorescence relative humidity (ERH) of inorganic salts used in this study. $\mathrm{N}_{\mathrm{i}}$ represents a neuron unit. $f_{\mathrm{ReLU}}$ and $f_{\text {Liner }}$ represent the activation function of ReLU and liner function, respectively. The detailed model parameters are listed in Table S2. 
In order to predict the ERH of inorganic salts used in this study, the predictive model was derived by using a neural network approach. Figure S3 illustrates the fundamental structure of the neural network employed in this study. The predictive model contains two hidden layers and one output layer. Each neuron unit, $\mathrm{N}_{\mathrm{i}}$, is calculated as,

$$
N_{i}=\sum_{1}^{j}\left(w_{j} \times \text { Input }_{j}\right)+\text { bias }_{i}
$$

52 Where $w_{j}$ is the weight factor, input $t_{j}$ is the input value from input or the previous layer's output, 53 and bias $_{i}$ is the residue in prediction. During the training process, both $w_{j}$ and bias $_{j}$ of each layer 54 are adjusted to fit the observation results.

In this study, the ReLU function is used for the activation function (Krizhevsky et al., 2012). The ReLU function is frequently used as the activation function to enhance nonlinear property in the neural network without a significant penalty for the generalization accuracy (Yarotsky, 2017). The number of neurons in the neural network determines the complexity of the model and impacts on its performance. In general, too few of neurons may result in underfitting that model cannot adequately capture the features from the input. However, too many neurons will cause overfitting which means the model memorizes the data itself and is not robust.

62

(a)

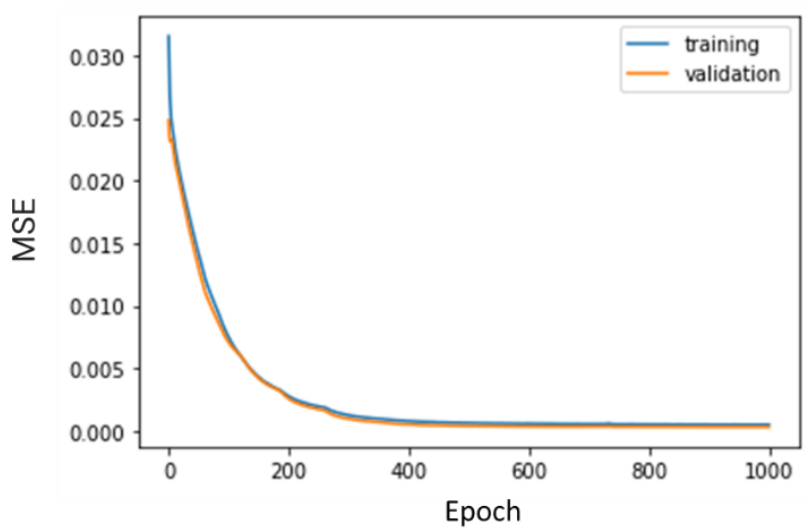

(b)

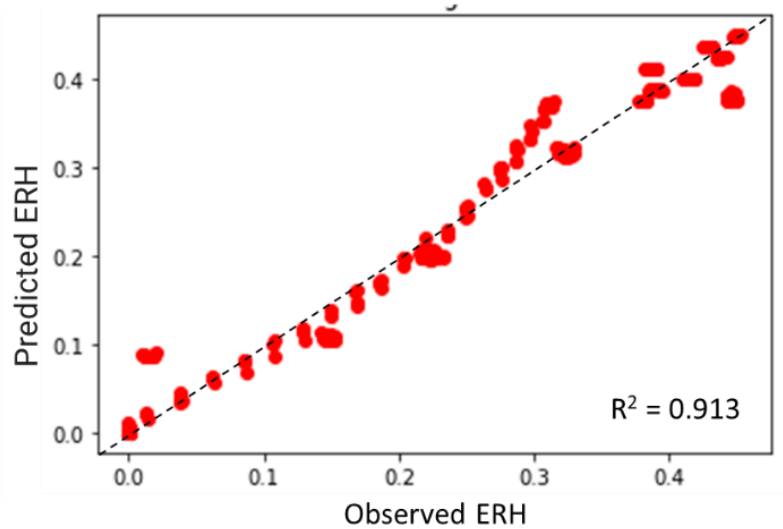

63

64

65

66

67
Figure S5. (a) The model performance for the training of deep neural network. (b) The predicted ERH using neural network model vs. observed ERH from literature values (Wu et al., 2019;Sun et al., 2018; Colberg et al., 2003). 
68 Table S3. Observations of efflorescence relative humidity of inorganic salts.

\begin{tabular}{ccccc}
\hline NO. & $\boldsymbol{f}_{\text {anion }}{ }^{\mathbf{a}}$ & $\boldsymbol{f}_{\text {Nitrate }} \mathbf{b}^{\mathbf{b}}$ & ERH & Reference \\
\hline 1 & 0.500 & 0.00 & 0.308 & Colberg, Christina A., et al. 2003 \\
2 & 0.500 & 0.00 & 0.285 & Colberg, Christina A., et al. 2003 \\
3 & 0.571 & 0.00 & 0.275 & Colberg, Christina A., et al. 2003 \\
4 & 0.667 & 0.00 & 0.160 & Colberg, Christina A., et al. 2003 \\
5 & 0.667 & 0.00 & 0.150 & Colberg, Christina A., et al. 2003 \\
6 & 0.800 & 0.00 & 0.000 & Colberg, Christina A., et al. 2003 \\
7 & 0.870 & 0.00 & 0.000 & Colberg, Christina A., et al. 2003 \\
8 & 1.000 & 0.00 & 0.000 & Colberg, Christina A., et al. 2003 \\
9 & 0.500 & 0.00 & 0.447 & Wu, Li, Xue Li, and Chul-Un Ro, 2019; Sun, Jiaxing, et al. 2018 \\
10 & 0.500 & 1.00 & 0.015 & Wu, Li, Xue Li, and Chul-Un Ro, 2019 \\
11 & 0.500 & 0.98 & 0.148 & Wu, Li, Xue Li, and Chul-Un Ro, 2019 \\
12 & 0.500 & 0.90 & 0.219 & Wu, Li, Xue Li, and Chul-Un Ro, 2019 \\
13 & 0.500 & 0.90 & 0.228 & Wu, Li, Xue Li, and Chul-Un Ro, 2019 \\
14 & 0.500 & 0.80 & 0.319 & Wu, Li, Xue Li, and Chul-Un Ro, 2019 \\
15 & 0.500 & 0.80 & 0.326 & Wu, Li, Xue Li, and Chul-Un Ro, 2019 \\
16 & 0.500 & 0.40 & 0.387 & Wu, Li, Xue Li, and Chul-Un Ro, 2019; Sun, Jiaxing, et al. 2018 \\
17 & 0.500 & 0.50 & 0.415 & Wu, Li, Xue Li, and Chul-Un Ro, 2019; Sun, Jiaxing, et al. 2018 \\
18 & 0.500 & 0.20 & 0.430 & Wu, Li, Xue Li, and Chul-Un Ro, 2019; Sun, Jiaxing, et al. 2018 \\
19 & 0.500 & 0.10 & 0.450 & Sun, Jiaxing, et al. 2018 \\
20 & 0.500 & 0.30 & 0.440 & Sun, Jiaxing, et al. 2018 \\
21 & 0.500 & 0.60 & 0.390 & Sun, Jiaxing, et al. 2018 \\
22 & 0.500 & 0.70 & 0.380 & Sun, Jiaxing, et al. 2018 \\
\hline
\end{tabular}

$70{ }^{\mathrm{b}} f_{\text {nitrate }}$ is the mole fraction of nitrate to total anion. 
72 Section S3. Aerosol phase state and phase transition for the SOA internally mixed with the

73 salted aqueous solution

74

75

76

77

78

79

80

81

82

83

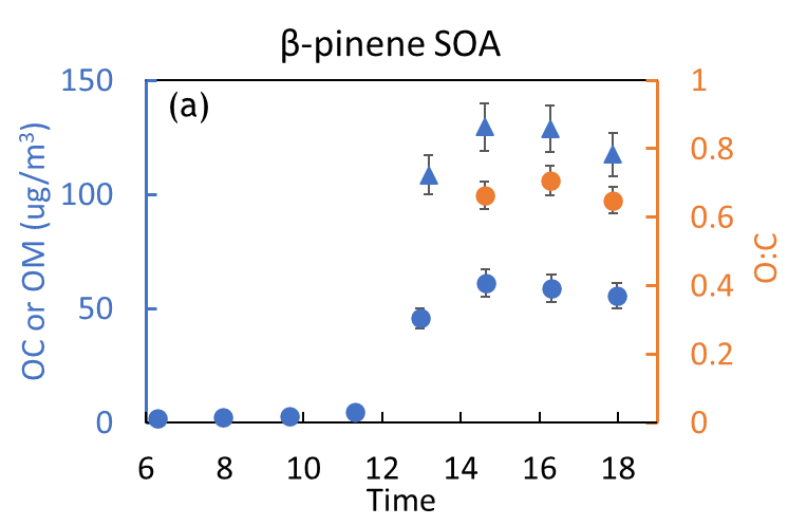

1,3,5-Trimethylbenzene SOA

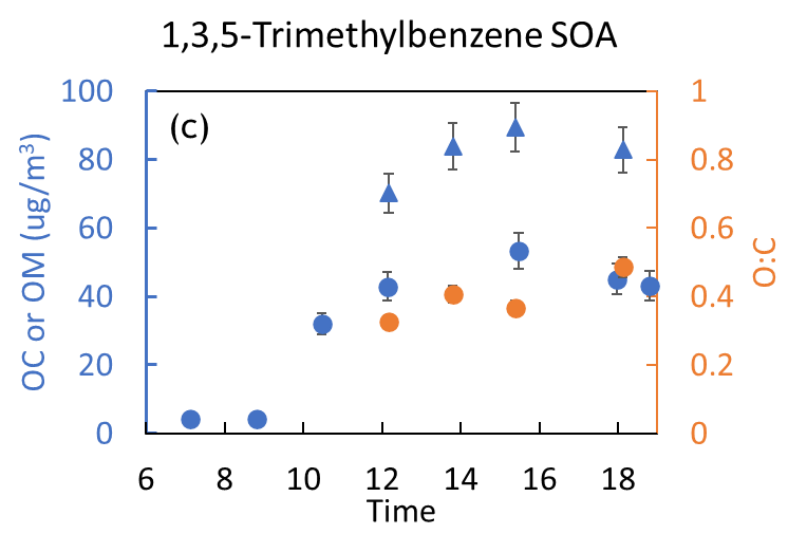
carbon atomic ratio.

where $\mathrm{OM}$ is the total organic mass concentration $\left(\mu \mathrm{g} \mathrm{m}^{-3}\right)$, $\mathrm{OC}$ is the organic carbon mass concentration $\left(\mu \mathrm{g} \mathrm{m}^{-3}\right), \mathrm{H}: \mathrm{C}$ is the hydrogen to carbon atomic ratio, and $\mathrm{N}: \mathrm{C}$ is the nitrogen to

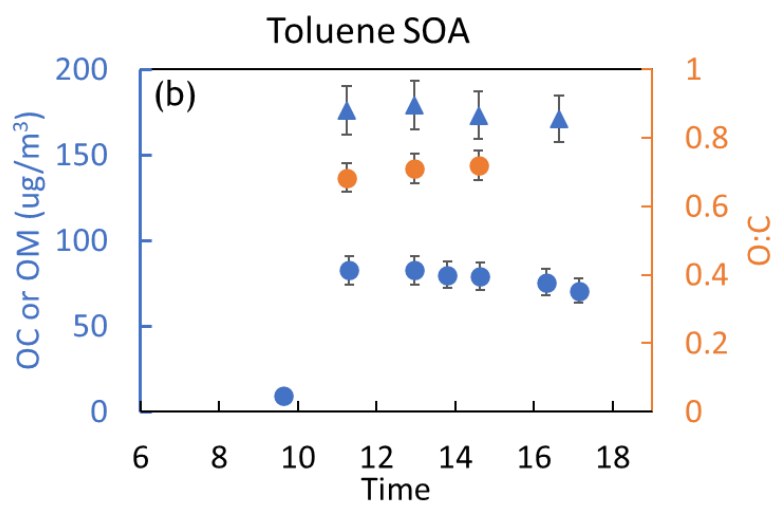

- OC measurement

$\triangle \quad$ OM measurement

- Estimated O:C ratio

85 Figure S6. The experimentally measured OM, OC and O:C ratios for (a) $\beta$-pinene SOA, (b) toluene SOA, and (c) 1,3,5-trimethylbenzene SOA. 
Table S4. The experimental conditions and model parameters used for estimating SRH of the SOA internally mixed with salted 88 aqueous solution.

\begin{tabular}{|c|c|c|c|c|c|c|c|c|}
\hline \multirow{2}{*}{ Precursor } & \multirow{2}{*}{ Date } & \multirow{2}{*}{$\begin{array}{c}\text { Sampling } \\
\text { time }\end{array}$} & \multirow{2}{*}{$\begin{array}{c}\text { Measured } \\
\mathrm{O}: \mathrm{C}^{\mathrm{a}}\end{array}$} & \multicolumn{3}{|c|}{ Organic parameters ${ }^{\mathrm{b}}$} & \multicolumn{2}{|c|}{ Inorganic parameters ${ }^{c}$} \\
\hline & & & & $\mathrm{MW}(\mathrm{g} / \mathrm{mol})$ & $\mathrm{H}_{\text {bond }}$ & Reference & IOR & $f_{\text {anion }}$ \\
\hline$\beta$-pinene & 03/04/2021-E & $\begin{array}{l}14: 36 \\
16: 16 \\
17: 52 \\
\end{array}$ & $\begin{array}{l}0.62-0.69 \\
0.65-0.74 \\
0.61-0.68 \\
\end{array}$ & $220-300$ & $0.32-0.48$ & $\begin{array}{c}\text { Table } S 1 \text { from } \\
\text { Yu et al., } 2021\end{array}$ & $0.2-0.8$ & $0.5-0.8$ \\
\hline toluene & 03/14/2021-E & $\begin{array}{l}11: 14 \\
12: 58 \\
14: 58 \\
\end{array}$ & $\begin{array}{l}0.63-0.72 \\
0.66-0.75 \\
0.67-0.76 \\
\end{array}$ & $220-300$ & $1.66-1.99$ & $\begin{array}{c}\text { Table } 1 \text { from } \\
\text { Zhou et al., } \\
2019 \\
\end{array}$ & $0.2-0.8$ & $0.5-0.8$ \\
\hline $\begin{array}{l}1,3,5- \\
\text { trimethylb } \\
\text { enzene }\end{array}$ & $03 / 14 / 2021-\mathrm{W}$ & $\begin{array}{l}12: 10 \\
13: 48 \\
15: 23 \\
18: 07 \\
\end{array}$ & $\begin{array}{l}0.30-0.34 \\
0.38-0.43 \\
0.34-0.38 \\
0.45-0.51 \\
\end{array}$ & $200-350$ & $0.1-0.45$ & $\begin{array}{c}\text { Table } 1 \text { from } \\
\text { Han and Jang, } \\
2020\end{array}$ & $0.2-0.8$ & $0.5-0.8$ \\
\hline
\end{tabular}

${ }^{a}$ The O:C ratio of SOA was calculated as described in Eq. S2.

$90 \mathrm{~b}^{\mathrm{b}}$ The possible ranges of $M W$ and $H_{\text {bond }}$ were obtained from the maximum and minimum values of the simulations that was reported in 91 reference studies (Yu et al., 2021;Han and Jang, 2020;Zhou et al., 2019). Briefly, SOA is simulated by using the UNIPAR model

92 which predicts the photochemically oxygenated products into 51 lumping groups based on volatility and aerosol phase reactivity. $M W$

93 and $H_{\text {bond }}$ are averaged by product distribution during the simulated time and then applied to this study.

$94{ }^{\mathrm{c}}$ The chamber generated SOA was collected on the filter and extracted with a solvent (water+methanol, 1:1 volume mix). The 95 resulting organic matter was blended with ammonium hydrogen sulfate aqueous solution. 


\section{Reference}

Berkemeier, T., Ammann, M., Mentel, T. F., Pöschl, U., and Shiraiwa, M.: Organic Nitrate Contribution to New Particle Formation and Growth in Secondary Organic Aerosols from a-Pinene Ozonolysis, Environ Sci Technol, 50, 6334-6342, 10.1021/acs.est.6b00961, 2016.

Bertram, A. K., Martin, S. T., Hanna, S. J., Smith, M. L., Bodsworth, A., Chen, Q., Kuwata, M., Liu, A., You, Y., and Zorn, S. R.: Predicting the relative humidities of liquid-liquid phase separation, efflorescence, and deliquescence of mixed particles of ammonium sulfate, organic material, and water using the organic-to-sulfate mass ratio of the particle and the oxygen-to-carbon elemental ratio of the organic component, Atmos. Chem. Phys., 11, 10995-11006, 10.5194/acp-11-10995-2011, 2011.

Canagaratna, M. R., Jimenez, J. L., Kroll, J. H., Chen, Q., Kessler, S. H., Massoli, P., Hildebrandt Ruiz, L., Fortner, E., Williams, L. R., Wilson, K. R., Surratt, J. D., Donahue, N. M., Jayne, J. T., and Worsnop, D. R.: Elemental Ratio Measurements of Organic Compounds using Aerosol Mass Spectrometry: Characterization, Improved Calibration, and Implications, Atmos Chem Phys, 15, 253-272, 10.5194/acp-15-253-2015, 2015.

Colberg, C. A., Luo, B. P., Wernli, H., Koop, T., and Peter, T.: A novel model to predict the physical state of atmospheric $\mathrm{H}_{2} \mathrm{SO}_{4} / \mathrm{NH}_{3} / \mathrm{H}_{2} \mathrm{O}$ aerosol particles, Atmos Chem Phys, 3, 909-924, DOI 10.5194/acp-3-909-2003, 2003.

Losey, D. J., Ott, E.-J. E., and Freedman, M. A.: Effects of High Acidity on Phase Transitions of an Organic Aerosol, The Journal of Physical Chemistry A, 122, 3819-3828, 10.1021/acs.jpca.8b00399, 2018.

Marcolli, C., and Krieger, U. K.: Phase Changes during Hygroscopic Cycles of Mixed Organic/Inorganic Model Systems of Tropospheric Aerosols, The Journal of Physical Chemistry A, 110, 1881-1893, 10.1021/jp0556759, 2006.

Nah, T., Sanchez, J., Boyd, C. M., and Ng, N. L.: Photochemical Aging of $\alpha$-pinene and $\beta$-pinene Secondary Organic Aerosol formed from Nitrate Radical Oxidation, Environ Sci Technol, 50, 222-231, 10.1021/acs.est.5b04594, 2016.

O'Brien, R. E., Wang, B. B., Kelly, S. T., Lundt, N., You, Y., Bertram, A. K., Leone, S. R., Laskin, A., and Gilles, M. K.: Liquid-Liquid Phase Separation in Aerosol Particles: Imaging at the Nanometer Scale, Environ Sci Technol, 49, 4995-5002, 10.1021/acs.est.5b00062, 2015.

Sun, J., Liu, L., Xu, L., Wang, Y., Wu, Z., Hu, M., Shi, Z., Li, Y., Zhang, X., and Chen, J.: Key role of nitrate in phase transitions of urban particles: implications of important reactive surfaces for secondary aerosol formation, Journal of Geophysical Research: Atmospheres, 123, 1234-1243, 2018.

Wu, L., Li, X., and Ro, C.-U.: Hygroscopic behavior of ammonium sulfate, ammonium nitrate, and their mixture particles, Asian Journal of Atmospheric Environment, 13, 196-211, 2019.

Yarotsky, D.: Error bounds for approximations with deep ReLU networks, Neural Networks, 94, 103-114, 2017.

You, Y., Renbaum-Wolff, L., and Bertram, A. K.: Liquid-liquid phase separation in particles containing organics mixed with ammonium sulfate, ammonium bisulfate, ammonium nitrate or sodium chloride, Atmos. Chem. Phys., 13, 11723-11734, 10.5194/acp-1311723-2013, 2013. 
142 Zhao, D. F., Schmitt, S. H., Wang, M. J., Acir, I. H., Tillmann, R., Tan, Z. F., Novelli, A., Fuchs, 143 H., Pullinen, I., Wegener, R., Rohrer, F., Wildt, J., Kiendler-Scharr, A., Wahner, A., and Mentel, T. F.: Effects of NOx and SO2 on the secondary organic aerosol formation from photooxidation of alpha-pinene and limonene, Atmos Chem Phys, 18, 1611-1628, 